\title{
Strengthening the emergency referral system in Cambodia for women and children under five: a description of interventions and impact analysis
}

\author{
Peter C Acker ${ }^{1}$, Loretta N Matheson ${ }^{1}$, Tuon Sovanna², Dim Sophearom², Matthew C Strehlow ${ }^{1}$ \\ 1 Department of Emergency Medicine, Stanford University School of Medicine, Palo Alto, California, USA, ${ }^{2}$ University Research Co. LLC, Phnom Penh, \\ Cambodia \\ Keywords: global health
}

https://doi.org/10.29392/joghr.3.e2019079

\section{Journal of Global Health Reports}

Vol. 3, 2019

\begin{abstract}
Background
Many factors align to reduce access to quality emergency services in Cambodia, resulting in unnecessary morbidity and mortality among the country's most vulnerable populations. The lack of a well-coordinated emergency referral system acts as a critical barrier for many seeking emergency care, limiting overall referral frequency and quality. To increase the effectiveness of the Cambodian healthcare system, the Quality Health Services (QHS) project implemented a spectrum of targeted, low-cost, locally-tailored interventions, including: triage systems, provincial clinical guidelines and referral hotlines, standardized referral-communication slips, and referral-feedback forms. To inform future health-system-strengthening efforts, the primary aim of this study is to describe the challenges facing the Cambodian referral system and the interventions applied to address these challenges. Secondarily, this study aims to characterize the frequency of emergency referrals in the post-intervention period and the utilization patterns of the implemented referral-system-strengthening tools.
\end{abstract}

\section{Methods}

Data was collected prospectively on a quarterly basis at 35 government referral hospitals in six Cambodian provinces between March 2016 and June 2017, data from 25 of these facilities was analyzed. Data on overall numbers of referrals was collected from each facility's referral logbook. The frequency and thoroughness of standardized referral-communication slip and standardized referral-feedback form use was ascertained by reviewing copies of these tools collected at each facility during the preceding quarter.

\section{Results}

The average number of emergency referrals to target facilities increased from 114.4 to 190.4 per quarter, a $66 \%$ increase. Target facilities also received $86 \%$ more standardized referral-communication slips and sent $200 \%$ more standardized referral-feedback forms per quarter during the monitoring period.

\section{Conclusions}

After receiving a package of referral-system-strengthening interventions the 25 hospitals analyzed in this study noted a significant increase in the frequency of emergency referral receipt. The frequency and quality of referral related communication improved consistently during the observation period as well. This data suggests that the interventions implemented addressed ongoing gaps in the Cambodian health system, and improved access to emergency referrals. These developments create a foundation upon which to build future health system strengthening interventions can be built and may provide guidance to implementors in other similarly resourced settings.

During the late 1970's, under the rule of the Khmer Rouge, the Cambodian medical system was dismantled, and the country's health workforce decimated. Although the Khmer Rouge were ousted from Phnom Penh in 1979, violence and political unrest continued for many years, drain- ing aid resources and hindering reconstruction efforts. As a result, during the 1980's and early 1990's, the country suffered some of the worst health outcomes in the world. ${ }^{1}$ Correspondingly, with the overall function of the health system being significantly depressed, initial multilateral develop- 
ment interventions yielded truly remarkable gains. In the period between 1979 and 2010 life expectancy at birth climbed from 22 to 66 years ${ }^{2}$, infant mortality rates fell from 128.4 to 37.8 per 1,000 live births ${ }^{3}$, and under-five mortality rates declined from 204 to 44 per 1,000 live births. ${ }^{4}$ The maternal mortality ratio was intermittently recorded prior to 1990, however dropped from 1020 in 1990 to 202 per 100,000 live births in $2010 .^{5}$

However, as development efforts matured, the remaining challenges have become more complex and difficult to address. As a result, advancements have slowed, and progress has plateaued. For the 5-year period between 2010 and 2015, as compared to the period between 1995 and 2010, there was a significant deceleration in the improvement rate for infant mortality, under- 5 mortality, and maternal mortality. 3,3,5

Although development efforts have helped to re-establish many essential components of the health system in Cambodia, having achieved or nearly achieved almost all of the health-related Millennium Development Goals ${ }^{6}$, access to quality emergency care remains critically limited for large portions of the population. Recently published data demonstrated that those living in low- and middle-incomecountries experience 8.6 million deaths that could have been averted had appropriate health care been provided. Of these $41.6 \%$ occur due to non-utilization of existing health care resources, while $58.3 \%$ occur due to the use of poorquality health services. ${ }^{7}$ This data suggests that challenges exist surrounding not only accessing available care, but provision of quality care when care can be reached. These conclusions appear applicable to Cambodia, where barriers to accessing appropriate, high quality emergency care are numerous and encountered at each step in the process.

While nearly $80 \%$ of the country's population resides in rural areas with poor transportation infrastructure, reaching a care facility is often a lengthy and costly process. ${ }^{8} \mathrm{Al}-$ though ambulances exist, they lack coordination, are typically staffed by only a driver (not an emergency care provider), and are often prohibitively expensive. ${ }^{9}$ These factors together lead to significant delays in seeking and reaching care. Going further, unfortunately, many Cambodian healthcare providers lack adequate emergency-specific skills and training, and as a result often fail to utilize the tools required to recognize and manage complex obstetric, pediatric, and general emergencies. ${ }^{10}$

Due to the limited supply of specialists and facilities with the capacity to care for life-threatening conditions, emergent patient referrals are often imperative. However, the Cambodian emergency referral system is difficult to navigate and inconsistently available. Insufficient referral-specific guidelines leave the process of selecting an appropriate receiving facility to the referring provider's best judgment, and as a result under- and over-triage is common. Also, without a structured referral communication system, communication of critical patient information from referring providers to ambulance staff and receiving providers is sporadic, leading to gaps and delays in care. ${ }^{11}$ With only a small number of facilities having adopted referral qualityimprovement and feedback measures, challenges are slow to be identified and addressed.
These system-based barriers drive down the overall numbers of patients obtaining emergency transport and receiving timely, appropriate referrals. As evidence, in assessments of unscheduled adult and pediatric hospital visits, although more than a third of adult patients and a quarter of pediatric patients presented as referrals from other facilities, only $9.3 \%$ and $2.9 \%$ (respectively) arrived by ambulance. ${ }^{10,12}$ The majority of these patients arrived at health facilities via private vehicle, taxi, non-motorized vehicle, or on foot. Additionally, the Cambodian Emergency Obstetric and Neonatal Care (EmONC) improvement plan 2010-2015 shows that in 2014 less than $25 \%$ of patients with obstetric complications delivered at EmONC facilities (the goal is $100 \%$ ), while a mere $0.3 \%$ of newborns were being referred from health centers to a higher level of care, though at least $5 \%$ would be expected. ${ }^{13}$ The lack of a functional emergency referral system remains a key contributor to the ongoing health disparities in Cambodia.

Fortunately, a variety of applicable interventions for strengthening referral systems have been trialed in other low-resource settings and have proven to be impactful. In India, the implementation of a free, centrally-coordinated, toll-free-number accessed ambulance service improved rates of institutional delivery. ${ }^{14}$ In Burundi, efforts to strengthen the referral system by improving referral communication systems, enhancing ambulance access, and promoting use of care guidelines were associated with a decreased risk of neonatal mortality. ${ }^{15}$ This study also noted significantly higher mortality rates for referrals taking $>3$ hours, reinforcing the time-sensitive nature of the process. In further support, a 2011 systematic review found that the most impactful maternal-health improvement programs included referral-system-strengthening components. ${ }^{16}$ Similarly, systematic reviews of the trauma literature have also noted significant morbidity and mortality benefits stemming from a variety of efforts to improve the accessibility, coordination, and function of emergency referral and transport systems. ${ }^{17,18}$ These international examples suggest that strengthening the referral system in Cambodia is an efficacious means by which to increase access to quality emergency care and improve health outcomes for a spectrum of vulnerable patient populations.

Efforts to improve access to emergency services within Cambodia have also shown promise. In a 2003 study, Husum et al. ${ }^{19}$ found that implementing a series of interventions to train and connect a network of rural Cambodian prehospital care providers had a significant positive impact on local trauma-related mortality. The same research group carried out a series of trainings to enhance traditional Cambodian birth attendants' ability to recognize and refer pregnant and postpartum women exhibiting danger signs, and noted a corresponding reduction in both maternal and neonatal deaths. ${ }^{20}$

Previous assessments into the feasibility and potential impact of referral-system-strengthening efforts in Cambodia found strong support among Cambodians, many of whom are frustrated with their current level of access to appropriate care and are interested in seeing the referral system's reach expanded. ${ }^{21}$ Prior work has also noted that although the system resources are constrained, progress is feasible by using innovative approaches to maximize the 
impact of current resources. Nakahara et al. performed a review in 2009 of the Cambodian trauma system and its resources noting:

\begin{abstract}
"Establishment of a formal ambulance system, although it is the long-term goal, is not realistic in the short term because of limited resources.” Instead "Streamlining referral mechanisms will require organization of each component of the referral mechanism by strengthening the existing system and mobilizing local resources, which would allow Cambodia to develop an efficient system at reasonable cost..... ${ }^{22}$
\end{abstract}

Fortunately, the country already possesses many key infrastructure items that, if leveraged appropriately, could create a functional, cost-effective referral system. ${ }^{23}$ For example: the road system has undergone significant improvements and plans for further expansion are in place 24,25 access to cell phone technology is nearly universal ${ }^{26}$, and the government already controls over 350 functioning ambulances. This number of ambulances provides for a ratio of one for every 40,000 Cambodian citizens, exceeding the World Health Organization's (WHO) recommendation of at least one ambulance for every 50,000 people. ${ }^{23}$ To spur further progress and enhance the quality of care, it is essential to improve coordination of these resources.

The United States Agency for International Development (USAID) sponsored Quality Health Service (QHS) project sought to improve the health outcomes of vulnerable populations (specifically maternal, newborn and pediatric) in Cambodia. In order to attain this goal, the project selected a number of high yield areas, including a focus on improving timely access emergency referrals.

The primary aim of this study is to describe the ongoing challenges facing Cambodia's referral system and the package of referral-system-strengthening interventions that were designed and implemented to address them. This study will detail the factors that led to the inclusion of each component, the experience and literature that supported the creation process, the implementation methods, and ongoing monitoring and evaluation measures with the hope of informing future health-system-strengthening efforts in related fields. Secondarily, this study aims to characterize both the overall frequency of emergency patient referral in the post-intervention period and the utilization of the implemented system-strengthening tools, which provide inferences to the overall uptake and impact of the interventions.

\section{CHALLENGES FACING CAMBODIAN REFERRAL SYSTEM - AND INTERVENTIONS APPLIED}

With improvement opportunities noted at each level of the referral system, the QHS project's implementers worked to design and disseminate a package of systems-based, referral-strengthening interventions responsive to the ongoing issues. Each of these items are described in detail below.

\section{VARIABLE RECOGNITION OF EMERGENCY CONDITIONS}

Unfortunately, due to training limitations, many Cambodian practitioners are not sufficiently equipped to recognize and manage acutely ill patients. At the same time, few if any Cambodian hospitals have systems in place to assist in identifying and prioritizing ill patients as they arrive. ${ }^{10}$ At many facilities, patients self-triage to the hospital department they deem most appropriate (eg, emergency department, intensive care unit, inpatient wards), where they are cared for in the order of arrival, delaying the care of critically ill patients and leading to worsened outcomes. With this in mind, as part of the project's efforts, a simple Cambodia-specific triage system was designed and implemented at 35 referral hospitals.

\section{NON-STANDARD TREATMENT AND REFERRAL OF EMERGENTLY ILL PATIENTS}

Although the Cambodia Ministry of Health (MOH) has created care guidelines for a number of conditions ${ }^{27-29}$, their guidelines are outdated, difficult to apply, and do not cover many key conditions. Also, they do not provide guidance as to when emergent referral is indicated, or how to coordinate and accomplish an emergent referral when it is required. In light of these notable gaps, QHS created and disseminated emergency care guidelines, tailored specifically to the Cambodian practice setting, for 73 specific maternal, pediatric, adult, and traumatic emergencies. 30

\section{INCONSISTENT ACCESS TO EXPERT CONSULTATION AND REFERRAL COORDINATION}

In resource-limited settings, effective communication systems are essential to the provision of quality emergency care. Functional communication systems allow experts at referral centers to share treatment guidance with providers at lower-level facilities. They also allow providers at the referring facilities to share important information about the patient and to efficiently coordinate referral logistics. Recognizing the role communication systems play in coordinating emergency care and referrals, many experts have highlighted the importance of including programs to enhance communication systems in broader health-systemstrengthening packages 23,31 and a variety of specific efforts to improve referral communications have been trialed. 15,32

Currently in Cambodia, communication between providers at various levels of the health system is uncommon, and relies on a fragmented, unreliable network of personal connections. ${ }^{22}$ In the rare instance that providers do seek to contact a higher-level facility for clinical advice or to arrange a referral, they typically do so by using their own personal cell phone to call the personal cell phone of a friend or contact at the target facility. Although occasionally successful, no redundancy is built into the system to account for change in phone numbers, times when the contact is off-duty, or their phone is out of power, and other potential complications. It is also rare that the contact is in fact the most appropriate person to reach for a given clinical re- 
quest, and subsequent calls are often required, further delaying care.

To increase the frequency of emergency referrals and referral-related communication, the QHS project established a series of referral hotlines. The pediatric, emergency, and maternity wards at each of the project's target hospitals received a dedicated phone. This phone was to be carried by the most senior clinician on duty at any given time and was to be accessible 24 hours a day. These phone numbers were distributed to all potential referring facilities within each referral hospital's catchment area, shared on an easy to read, hotline sheet (see Appendix S1 in the Online Supplementary Document). Providers at lower-level facilities were encouraged to use this hotline to seek input regarding the care of acutely ill patients, including guidance on the need for a referral and to efficiently establish referral logistics. Referral hospital staff were coached on optimal referral-communication strategies based on the project's referral guidelines.

\section{LACK OF TRAINED AMBULANCE-CARE PROVIDERS}

Currently in Cambodia, patients who do receive ambulance transport most often do so without the accompaniment of a healthcare provider. ${ }^{13}$ For the fortunate minority of patients who are accompanied by a provider during referral, it is unlikely the transporting provider has received any specific training in ambulance care.

Noting this key gap, the QHS project designed and implemented a modular curriculum focused on the most common and life-threatening pediatric, maternal, traumatic, and adult emergencies. During the project, this course was provided to over 900 providers at 38 referral hospitals within the project's target provinces. ${ }^{33}$

\section{INADEQUATE COMMUNICATION OF DATA COLLECTED AND TREATMENTS BY REFERRER}

Written referral slips are another essential means through which referral-related communication can be accomplished. Written communication is not only the most common form of referral communication in use, in many situations it is the only form used. ${ }^{34}$ Evidence suggests that using written referral slips increases the likelihood a given referral will be successfully completed ${ }^{11}$, and can improve the accuracy and reduce redundancy of care provided by receiving practitioners. ${ }^{35,36}$ The use of such communication tools is recommended by many expert groups and systems, including the WHO's Integrated Management of Childhood Illness program. ${ }^{37}$

Although these tools have significant potential, in many instances they are found to contain inadequate information, slowing necessary downstream care. ${ }^{38}$ However, the use of standardized referral forms can help increase the amount of valuable information transmitted in this manner. ${ }^{39,40}$

The Cambodia MOH had created and recommended the use of a standardized referral slip during all patient referrals 41 , however the form they created lacked adequate structure to promote quality information transfer, particularly around the details of care provided to maternity patients, pediatric patients, and neonatal patients. To increase the impact of this referral-communication tool, the project updated the referral slip to improve its ability to efficiently capture practice-changing information. Individual sections were inserted with checkboxes that could be used to quickly indicate the status of and treatments provided to each of these vulnerable patient groups. Prompts were also built into the form to remind referring providers to send auxiliary data sources, such as a partograph, along with the patients. The form was also made into a quadruplicate, which allowed copies to be easily distributed to all interested parties (see Appendix S2 in the Online Supplementary Document). Introduction of this new tool was paired with a number of educational and support interventions, which are detailed below.

\section{INADEQUATE COMMUNICATION ON DATA COLLECTED AND TREATMENTS IN AMBULANCE}

To improve communication related to ambulance care, project effort also went into updating the ambulance patient care report (PCR). The form was amended to promote recording of the timing of care and transport, as well as the names of the providers involved in ambulance care to allow for enhanced quality-improvement interventions.

\section{LACK OF FEEDBACK FROM RECEIVING TO REFERRING PROVIDER}

Provision of feedback on referred cases to referring providers represents a vital quality improvement opportunity. Referring providers can be supplied with coaching from receiving providers, receive updates on their patient's eventual treatment, diagnosis, and outcomes, and also be made aware of any ongoing treatment needs the patient may have going forward. Unfortunately few referring providers in resource-limited settings receive feedback on referred cases. ${ }^{42-44}$

Under this project, a more comprehensive, standardized referral-feedback form was created. Similar to the updates made to the standardized referral-communication slip, the feedback form was revised to improve efficiency of use, while promoting the recording of actionable feedback. The new form now contains sections to describe a patient's overall care course, eventual diagnosis, any specific followup care they will need at the referring facility, as well as a new section for specific constructive feedback to the referring provider. The form was also created in triplicate, with one copy being kept at the receiving facility, one being sent back to the referring facility with the patient, and one being sent back to the referring facility via the local governmentquality-improvement body (see Appendix S3 in the Online

\section{Supplementary Document).}

The aim of each of these interventions was to increase the frequency with which the referral system was used to rapidly connect patients suffering from emergencies with the care they required, while systematically increasing the quality of care being provided prior to, during, and after referral. 


\section{IMPLEMENTATION OF THE PROJECT INTERVENTIONS}

\section{INITIAL INTRODUCTION}

Rollout took place in a staggered manner between September 2015 and August 2017 (see Figure 1). QHS team members and $\mathrm{MOH}$ counterparts visited all referral hospitals within a selected province and conducted an initial twoday orientation. On the first day of the orientation, team members introduced the tools and corresponding workflows to hospital and ward leadership. Educators then met with providers on each ward, demonstrating tool use through practical application cases. On the second day of orientation, hospital staff began utilizing the tools with team member oversight and structured coaching. Ward staff were provided instruction and tools to promote regular data collection and assessment. Systems were collaboratively created to both provide referral feedback and communicate referral data within each facility and to provincial oversight groups.

\section{SUSTAINED SUPPORT}

Facilitators returned to each facility one month after orientation, and then on a quarterly basis to provide further education and coaching, troubleshoot ongoing challenges, and seek quality-improvement feedback. During follow-up visits, project staff worked with local staff to collect referral data, strengthen local data-collection systems, and summarize themes to communicate to hospital and provincial leadership. Quarterly support visits continued through September 2018.

\section{METHODS}

\section{TARGET AREA}

The referral-strengthening interventions were provided to 35 government-affiliated referral hospitals throughout nine Cambodian provinces: Banteay Meanchey, Battambang, Kampong Cham, Kampong Speu, Pailin, Prey Veng, Pursat, Siem Reap, and Tbong Khmum (see Figure 2). The total population of these nine provinces is 6.9 million, representing nearly half of the country's overall population. ${ }^{45}$ Note, within the Cambodian health system, all hospitallevel facilities are called "referral hospitals" because they receive referred patients; the lower-level facilities referring patients are typically health centers or lower-lever hospitals, referred to in this report as referring facilities.

\section{DATA COLLECTION}

Using project-specific tools, data was collected on a quarterly basis from the maternity, pediatric, and emergency wards at each facility. The analysis addresses data from the $1^{\text {st }}$ through $5^{\text {th }}$ follow-up visits at the 25 facilities that had three or more follow-up visits during the data-collection period (January 2016 and June 2017). Only six facilities had a $6^{\text {th }}$ follow-up visit; data from this time point was excluded.

The team collected data on referral frequency by examining the government-issued, ward-specific referral log book

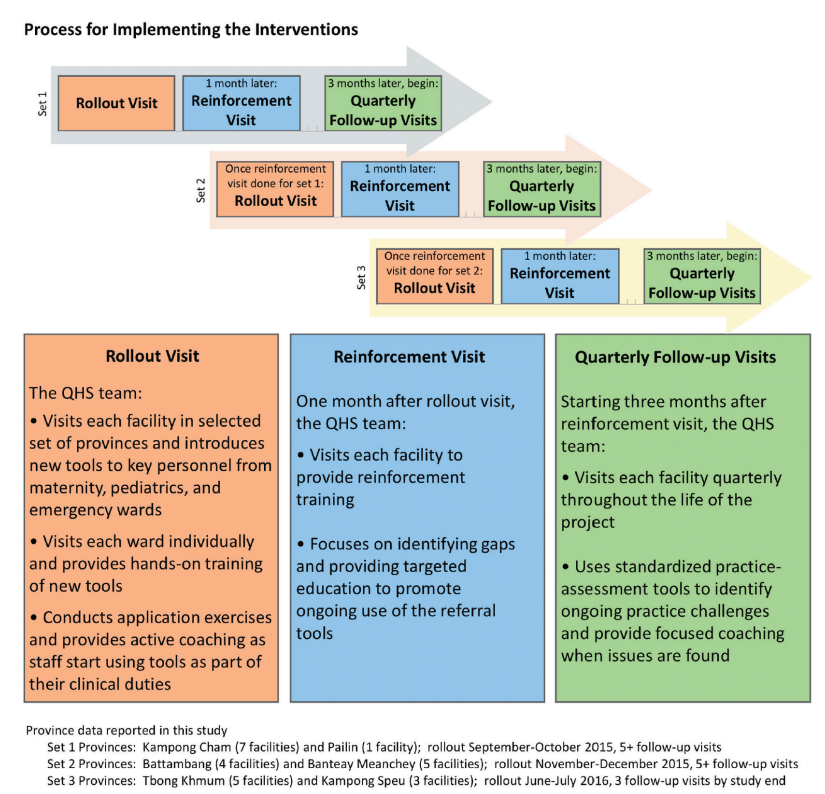

\section{Figure 1}

Intervention rollout and follow-up process.

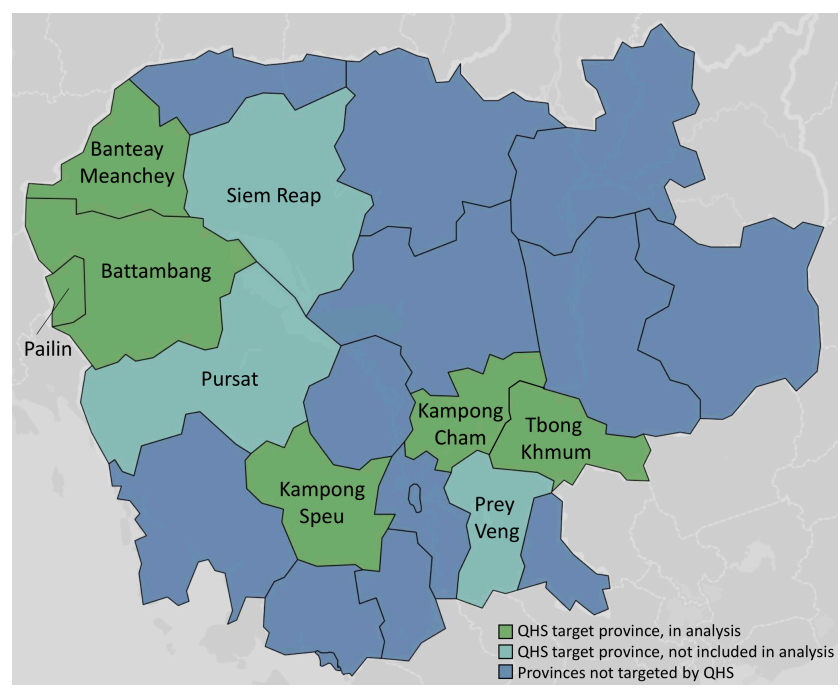

\section{Figure 2}

Map of Cambodia highlighting provinces included in QHS project and in data analysis.

at each facility. Facility staff are required by the government to record each referral in this logbook. With the introduction of referral-communication slips, there may have been confusion about whether to also enter those referrals into the log book. In follow-up visits where more referral slips were encountered than referral entries in the facility's log book, the number of referral slips was used as the overall number of referrals.

Data on the use of referral-system-strengthening tools were gathered by assessing the referral slips and feedback forms collected by staff at each facility during the preceding quarter. Total numbers of each form type received were recorded, along with the presence or absence of key pieces 
of data that were used as indicators of thoroughness of completion.

Data collected in the field by QHS team members was initially stored in Microsoft Excel (Microsoft Inc, Seattle, WA, USA). Data was then uploaded and stored in QHS' online data storage platform, Project Database System (PDS).

\section{STATISTICAL ANALYSIS}

SAS Enterprise Guide (SAS, Cary, NC, USA) version 7.15 was used for analysis. Tableau (Tableau, Seattle, WA, USA) version 10.4 was used for visualizations.

\section{RESULTS}

\section{OVERALL FREQUENCY OF REFERRALS}

During the assessment period, the average number of referrals received per facility per quarter increased by $66 \%$, from 114.4 to 190.4 . Overall, each ward type noted increases in quarterly patient referrals with the maternity ward, pediatric ward and emergency ward experiencing increases of $28 \%$ (66.8 to 85.2 ), $94 \%$ (8.7 to 16.9 ) and $127 \%$ (38.9 to 88.2 ), respectively (see Figure 3).

\section{FREQUENCY OF STANDARDIZED REFERRAL- COMMUNICATION SLIP USE}

Use of the standardized referral-communication slip increased by $86 \%$, with target facilities initially receiving 83.2 per quarter and receiving 154.6 per quarter by the end of the study period. Correspondingly, each ward type received increasing numbers of the slip throughout the assessment period. The maternity ward, pediatric ward and emergency ward noted increases of $29 \%$ (49.8 to 64 ), $211 \%$ (3.8 to 11.8 ) and $167 \%$ (29.5 to 78.9$)$, respectively (see Figure 3 ).

\section{COMPLETENESS OF THE STANDARDIZED REFERRAL- COMMUNICATION SLIPS}

Presence of contact details and a signature from the referring and receiving providers on the standardized referralcommunication slips were key indicators of thorough slip completion. During the study period, the frequency with which collected slips contained these key indicators improved. The frequency with which referring provider's information was recorded increased by $13 \%$, from $83 \%$ to $94 \%$ (average number of slips containing referrer information received per quarter increased from 72 to 150), while frequency with which the receiving provider's information was recorded increased by $43 \%$, from $51 \%$ to $73 \%$ (average number of slips containing receiver information received per quarter increased from 52 to 136) (see Figure 4).

\section{FREQUENCY OF STANDARDIZED REFERRAL-FEEDBACK FORM USE}

The frequency of cases for which standardized referralfeedback forms were completed by the receiving provider and sent back to the referring provider also rose. It was found that at the first follow-up visit, feedback was provided using the standardized referral-feedback form in approxi-

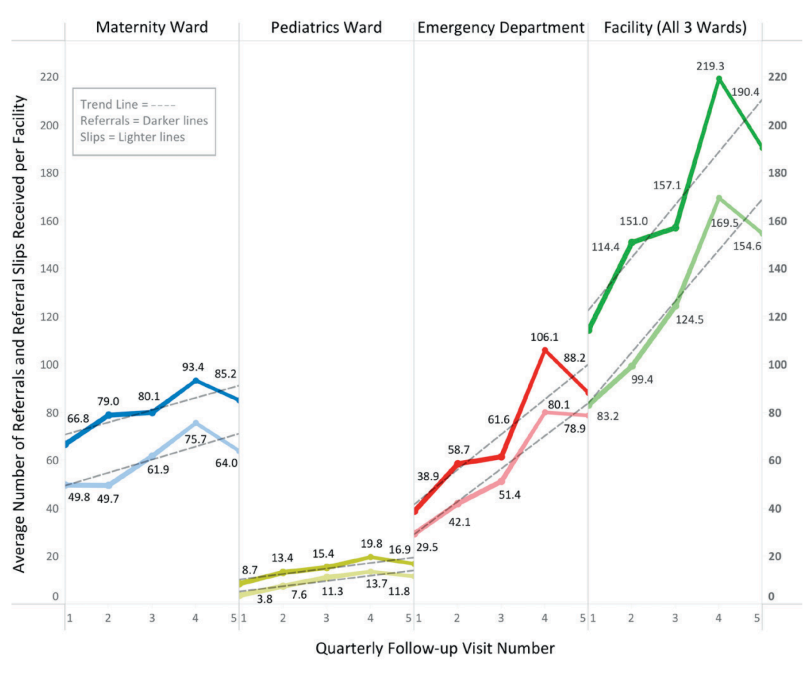

\section{Figure 3}

Average number of referrals and standardized referral-communication slips received per quarter (by ward and total by facility). This figure represents data from 25 target referral hospitals, collected on a quarterly basis from each facility's government-issued referral log books. During the assessment period, the overall number of patients referred into target facilities increased by $66 \%$ while the overall number of standardized referral-communication slips received by target facilities increased by $86 \%$. Increases of referrals and receipt of standardized referral-communication slips were also noted at the ward level for all of the assessed ward types (Maternity, Pediatric, and Emergency).

mately $13 \%$ of cases. By the fifth follow-up visit, standardized referral-feedback forms were used for $39 \%$ of cases, a net increase of $200 \%$ (average number of feedback forms sent increased from 17 to 41 per quarter) (see Figure 4).

\section{FREQUENCY OF PRE-REFERRAL NOTIFICATION CALLS}

The regularity with which referrals were preceded by a notification phone call from the referring provider to the receiving facility remained low throughout the study period. Initial rates were $6.9 \%$ and increased only slightly to $7.2 \%$ by the study's conclusion (average number of pre-referral notification calls received per quarter increased from 7 to 9) (see Figure 4).

\section{DISCUSSION}

\section{OVERALL REFERRAL-SYSTEM UTILIZATION}

During the monitoring period, the number of patients undergoing referral in the target area increased. This data suggests a temporal correlation between the implementation of QHS' referral-system-strengthening interventions and the increase in patient referrals. The utility of the referral-system-strengthening tools likely buoyed this trend, as did the consistent quarterly follow-up visits by project staff. It should be noted that during the final quarter, referrals received decreased across all ward types. The factors underlying this change are yet to be elucidated. Overall, although potential confounders exist, the increased number of emergency referrals suggests an improvement in the accessibility and function of the emergency referral system.

While referrals and standardized referral-communication slips usage increased across all ward types, the emer- 
gency ward saw particularly notable growth. And although the pediatric wards received relatively fewer referrals, they showed an overall increasing trend of use, more than doubling the number of standardized referral-communication slips received during the monitoring period.

The discrepant volume of pediatric referrals may be related to a few factors. First, relative to the pediatric population, there are significantly more emergency and maternity patients presenting to government health facilities in Cambodia. As such the potential for increases in referral numbers is far greater among the emergency ward and maternity ward patient populations. Second, intermittently at a number of facilities, pediatric referrals underwent their initial assessments in the emergency ward prior to the pediatric ward. At these facilities, some of the pediatric referrals were likely recorded instead as emergency ward referrals.

The increase in overall referral frequency noted in this study equates to just under 1 additional referral per facility per day, which represents significant progress. Patients selected to undergo emergency referral are chosen from a small subset of presenting patients, those with critical illness, for whom a referral may be the single most important factor in determining their outcome. Even a modest absolute increase in referrals among this group could have positive impact on health indicators.

As an example, Cambodia's maternal mortality ratio (MMR) was 161 per 100,000 live births in 2015, and 371,000 total live births were reported that year, thus 597 annual maternal deaths would have been expected throughout the country. 5,46 Based on the overall increase in maternal referrals noted during the observation period (66.8 to 85.2 per quarter), the average facility would expect to receive an additional 73.6 maternal referrals per year. Assuming the referred patients are from the sickest subset of those presenting to health facilities, and that the referral represents an opportunity to save a life that did not previously exist, even this small change could have a notable impact on the country's health outcomes, maternal and otherwise.

Similarly, although the overall change in the absolute numbers of patients being referred is modest, the positive trajectory of the change suggests that system-strengthening interventions are capable of yielding improved system function in Cambodia. Having now been extensively piloted and thoroughly revised, going forward these interventions can provide the foundation for ongoing referral system growth both within and outside the project's target provinces.

\section{UTILIZATION OF REFERRAL-SYSTEM-STRENGTHENING TOOLS}

Based on conversations with health leaders throughout the project's target area and interactions with the $\mathrm{MOH}$, it appears that support for QHS' referral-system-strengthening interventions has become widespread. Facility-based providers find the tools helpful, appropriate to their practice setting, and easy to use. The MOH has also lent their support, endorsing the standardized referral-communication slip, ambulance PCR and standardized referral-feedback form, now advocating for their dissemination and use at the national level.

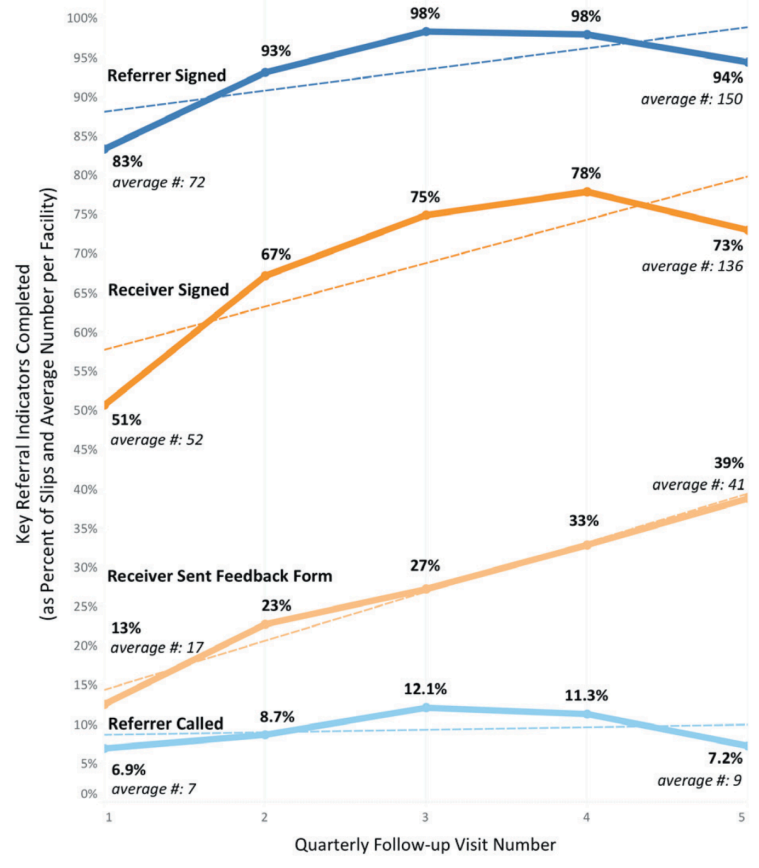

\section{Figure 4}

Performance of referral-system-strengthening process indicators. Data on the performance of a number of key referral-system-strengthening indicators was gathered from standardized referral-communication slips and standardized referral-feedback forms collected by facility staff on a quarterly basis. During the assessment period, use of the standardized referral-communication slip increased by $86 \%$, inclusion of the referring-provider's contact information on collected standardized referral-communication slip increased by $13 \%$, inclusion of the receiving-provider's contact information on the collected standardized referral-communication slips increased by $43 \%$, while use of the standardized referral-feedback forms increased by a net of $200 \%$. Pre-referral notification calls increased by $4 \%$.

\section{STANDARDIZED REFERRAL-COMMUNICATION SLIP USE}

Uptake of the standardized referral-communication slip occurred rapidly. By the third follow-up visit, slip use across wards had increased by an average of 50\%. Uptake within emergency wards caught on particularly quickly, increasing by $74 \%$ in the same period. This pattern suggests the form was found to be usable, and providers were motivated to use it.

Not only did use of referral slips increase overall, but the accuracy and completeness with which they were filled out also improved. As mentioned, the presence or absence of signatures and contact information from referring and receiving providers were used as a marker of completion. During the data collection period, the percent of slips bearing both indicators increased.

Rates of referring provider information were consistently higher than those of the receiving provider. The workflow established has the referring provider initially completing the majority of the referral slip, sending it with the referred patient to the receiving facility, where it is to be assessed and signed by the receiving provider. Field teams noted that with this workflow, the referring provider benefited from a number of reminders to complete the slip that the receiving provider lacked. This may explain a portion of the discrepancies in completion rates noted between the two groups. 
At a basic level, with increasing frequency of the receiving provider's signature suggests that receiving providers were viewing the patient referral information more frequently, suggesting the patient information transferred via the slips increased with time.

\section{STANDARDIZED REFERRAL-FEEDBACK FORM USE}

Before QHS, feedback on referrals was extremely rare and non-structured when it did occur. However, with the advent of a standardized feedback form and reinforcement of a consistent feedback provision system, by the fifth follow-up visit referring providers were receiving feedback on nearly $40 \%$ of referral-slip cases.

Unlike many of the other indicators assessed, use of the standardized referral-feedback forms did not decline in the final quarter of the assessment period, instead consistent progress was noted. Unlike many of the other indicators assessed, use of the standardized referral-feedback forms did not decline in the final quarter of the assessment period, instead consistent progress was noted. This intervention likely benefited from the support of a number of complementary, QHS-led quality-improvement efforts.

\section{PRE-REFERRAL NOTIFICATION CALLS}

Although most of QHS' referral-strengthening tools experienced strong uptake and appeared to impact referral practices, the creation of provincial clinical and referral hotlines did not appear to have an impact on the frequency of prereferral communication. Even with the support of the communication system created under QHS, an average of only $7 \%$ of referrals were preceded by a notification call to the receiving facility by the end of the assessment period; nearly all of these cases were maternal referrals.

Although issues with the data recording and collection process may have falsely lowered these numbers, a number of real-world barriers existed. Phones were lost, damaged, ran out of batteries, and numbers changed at times. Each of these issues created periods during which a hotline was inaccessible. Field interviews indicated that facility provider confidence in the hotline system declined each time they unsuccessfully attempted to access the hotline and decreased the likelihood of future use.

Another theme noted was a strong preference among facility staff to contact a familiar provider when making carerelated calls. Previously, when providers contacted higherlevel facilities, they would do so by calling an acquaintance at that facility. This collegial network afforded a feeling of safety and allowed calling providers to avoid scenarios in which they felt their knowledge was being scrutinized. The referral hotlines, however, often connected providers that were previously unfamiliar. As such, referring providers were concerned that their knowledge may be judged critically and were thus less likely to place pre-referral calls. Future work is required to craft systems-based solutions that account for this communication barrier.

\section{LIMITATIONS}

This study faced a number of limitations. Creation of all the raw data upon which this study was based required input from facility-based providers. Whether it was collecting and storing referral-communication slips and referral-feedback forms or noting referrals in a hospital's referral log book, all of the collected data points were reliant on provider effort. As such, in instances when providers did not complete one of the tasks required to capture data, those data points were lost. Although impossible to know how frequently this may have occurred, it is a possible source of underreporting.

As noted in the background section, the referral-systemstrengthening interventions were just one aspect of a much larger project aimed at improving outcomes for vulnerable populations in Cambodia. Though the other project components were not specifically focused on improving the function of the referral system, their efforts were likely complementary, and may have impacted referral practices at the health center and referral hospital level. It was not possible to delineate between the effects of the project's other interventions on referral trends and those of the above detailed referral-strengthening interventions.

Although this data provides insight into practice trends in the target provinces, it does not allow comparisons to be made against non-intervention provinces as the study lacks a control group. Data on referral practices in non-target provinces are collected and stored by the $\mathrm{MOH}$ but are not yet available. Similarly, the data available describing preintervention referral practices were found to be unreliable. As such, it was not possible to establish an accurate pre-intervention baseline; instead, data from the first post-intervention visit were used as the baseline for comparison.

Lastly, this analysis did not include an assessment of the quality-of-care recommendations provided through the communication system, nor the appropriateness of referrals being made. Although a number of measures were enacted to avoid unintended negative consequences, it is possible that inappropriate use of these systems had adverse effects that were not measured.

\section{CONCLUSIONS}

Under the USAID QHS project, comprehensive referral-system-strengthening interventions were implemented across 35 referral hospitals in Cambodia. These interventions were designed to both increase accessibility to and strengthen the function of the Cambodian referral system. Based on experience with emergency referral systems in Cambodia and other LMICs, the interventions focused specifically on increasing the quality of peri-referral care, improving referral-related communication, supporting ongoing qualityimprovement, and streamlining referral logistics by enhancing the function and coordination of currently existing referral system components.

The application of these interventions was correlated with an increase in the frequency of maternal, pediatric, and general emergency patient referrals within six Cambodian provinces. Overall use of the individual referral tools increased throughout the assessment period as well. These findings suggest that the project's efforts successfully in- 
creased the function of Cambodia's referral system, and as a result, access to emergency care for the country's most vulnerable populations.

Keys to success included the use of a comprehensive intervention-rollout education package, consistent follow-up visits to reinforce use of the tools, enhanced data-monitoring systems, and extensive collaboration with local systems experts. Although it is yet to be seen, it is hoped that the buy-in achieved during this project will lead to sustained use of these interventions and continued development of the Cambodian referral system.

Future efforts should focus on assessing the quality of care being provided by facilities having received the referral-strengthening interventions, as well as the appropriateness of the referrals being made. Use of appropriate controls, including data assessment from provinces outside of the project's target area will help to further clarify the impact of these quality-improvement measures.

\section{ACKNOWLEDGEMENTS}

Our gratitude goes to Dr. Sok Srun, the Deputy Director of the Cambodian Ministry of Health's Department of Hospital Services, for his input and strong support of the referralsystem-strengthening efforts. We also thank our numerous government health system colleagues within the Ministry of Health, the Provincial Health Departments and each of the referral hospitals in the nine target provinces, whose work will help sustain the gains made during this project into the future. We would like to thank Dr. Som Hun (QHS Chief of Party) and Dr. Katherine Krasovec (former QHS Chief of Party), for their expertise and leadership in the project's de- sign and implementation, and Dr. Iv Ek Navapol (QHS Chief of Monitoring and Evaluation) for his assistance and the assistance of his team with data collection and sharing. We would like to thank Mr. Sao Vuthoeun (QHS team member), and Mr. Va Pisal (previous QHS team member) for their diligence in data collection and provider education. We would also like to thank Dr. Jennifer Newberry and Dr. Mike Kohn for their input and expertise on data analysis and design.

\section{FUNDING}

The research described in this manuscript was performed as part of the Quality Health Services project, which was funded by USAID.

\section{COMPETING INTERESTS}

The authors completed the Unified Competing Interest form at http://www.icmje.org/coi disclosure.pdf (available upon request from the corresponding author), and declare no conflicts of interest.

\section{CORRESPONDENCE TO:}

Peter C Acker MD/MPH, FACEP

Assistant Professor of Emergency Medicine

Department of Emergency Medicine

Stanford University School of Medicine

900 Welch Road, Suite 350

Palo Alto, California, 94304

USA

packer@stanford.edu 


\section{REFERENCES}

1. Cambodia World Bank Development Indicators Graphical Overview. Accessed August 21, 2018. http s://data.worldbank.org/country/cambodia

2. Cambodia World Bank Development Indicators - Life Expectancy at Birth. Accessed October 28, 2017. http s://data.worldbank.org/indicator/SP.DYN.LE00.IN?loc ations $=\mathrm{KH}$

3. Cambodia World Bank Development Indicators Infant Mortality Rate. Accessed August 28, 2018. http s://data.worldbank.org/indicator/SP.DYN.IMRT.IN?loc ations $=\mathrm{KH}$

4. Cambodia World Bank Development Indicators Under-5 Mortality Rate. Accessed August 28, 2018. htt ps://data.worldbank.org/indicator/SH.DYN.MORT?loc ations $=\mathrm{KH}$

5. Cambodia World Bank Development Indicators Maternal Mortality Ratio. Accessed August 28, 2018. ht tps://data.worldbank.org/indicator/SH.STA.MMRT.N E?locations $=\mathrm{KH}$

6. Ministry of Planning. Annual Progress Report: Achieving the Millennium Development Goals. Royal Government of Cambodia Ministry of Planning; 2013. Accessed September 17, 2018. http://www.undp.org/c ontent/dam/undp/library/MDG/english/MDG\%20Cou ntry\%20Reports/Cambodia/CMDG\%20Annual\%20Rep ort\%202013 Final\%20Draft.pdf

7. Kruk ME, Gage AD, Joseph NT, Danaei G, GarcíaSaisó S, Salomon JA. Mortality due to low-quality health systems in the universal health coverage era: a systematic analysis of amenable deaths in 137 countries. Lancet. 2018;392(10160):2203-2212. doi:1 0.1016/s0140-6736(18)31668-4

8. Cambodia World Bank Development Indicators Percent Rural Population.; 2015. Accessed August 28, 2018. http://data.worldbank.org/indicator/SP.RUR.TO TL.ZS?locations $=\mathrm{KH}$

9. Sasakawa Peace Foundation. Study Report on Emergency Medical Care in Cambodia [Internet]. The Sasakawa Peace Foundation; 2011. Accessed July 19, 2018. https://www.spf.org/dbfiles/27940d80aa0.pdf

10. Yan LD, Mahadevan SV, Yore M, et al. An observational study of adults seeking emergency care in Cambodia. Bull World Health Organ.

2015;93(2):84-92. doi:10.2471/blt.14.143917
11. Kalter H, Salgado R, Moulton L, et al. Factors constraining adherence to referral advice for severely ill children managed by the Integrated Management of Childhood Illness approach in Imbabura Province, Ecuador. Acta Paediatr. 2007;92(1):103-110. doi:10.11 11/i.1651-2227.2003.tb00478.x

12. Yore MA, Strehlow MC, Yan LD, et al. Characteristics and outcomes of pediatric patients presenting at Cambodian referral hospitals without appointments: an observational study. Int J Emerg Med. 2018;11(1). doi:10.1186/s12245-018-0172-0

13. Bunsoth M, Middleton J, Vonthanak S, Aim S, Hou S. Review of the Cambodia Emergency Obstetric and Newborn Care Improvement Plan 2010-2015. MBS Research Team; 2015.

14. Prinja S, Jeet G, Kaur M, Aggarwal AK, Manchanda N, Kumar R. Impact of referral transport system on institutional deliveries in Haryana, India. Indian J Med Res. 2014;139:883-891.

15. Tayler-Smith K, Zachariah R, Manzi M, et al. An ambulance referral network improves access to emergency obstetric and neonatal care in a district of rural Burundi with high maternal mortality. Trop Med Int Health. 2013;18(8):993-1001. doi:10.1111/tmi.121 $\underline{21}$

16. Nyamtema AS, Urassa DP, van Roosmalen J. Maternal health interventions in resource limited countries: a systematic review of packages, impacts and factors for change. BMC Pregnancy Childbirth. 2011;11(1):30. doi:10.1186/1471-2393-11-30

17. Henry JA, Reingold AL. Prehospital trauma systems reduce mortality in developing countries: A systematic review and meta-analysis. J Trauma Acute Care Surg. 2012;73(1):261-268. doi:10.1097/ta.0b013e 31824bde1e

18. Reynolds TA, Stewart B, Drewett I, et al. The impact of trauma care systems in low- and middleincome countries. Annu Rev Public Health. 2017;38(1):507-532. doi:10.1146/annurev-publhealt $\underline{\mathrm{h}-032315-021412}$

19. Husum H, Gilbert M, Wisborg T, Van Heng Y, Murad M. Rural prehospital trauma systems improve trauma outcome in low-income countries: A prospective study from North Iraq and Cambodia. $J$ Trauma. 2003;54(6):1188-1196. doi:10.1097/01.ta.000 0073609.12530 .19 
20. Houy C, Ha SO, Steinholt M, Skjerve E, Husum H. Delivery as trauma: A prospective time-cohort study of maternal and perinatal mortality in rural Cambodia. Prehosp Disaster Med. 2017;32(2):180-186. doi:10.1017/s1049023x1600145x

21. Jacobs B, Men C, Sam OS, Postma S. Ambulance services as part of the district health system in lowincome countries: a feasibility study from Cambodia. Int J Health Plann Manage. 2016;31(4):414-429. doi:1 $\underline{0.1002 / \mathrm{hpm} .2285}$

22. Nakahara S, Saint S, Sann S, et al. Exploring referral systems for injured patients in low-income countries: a case study from Cambodia. Health Policy Plan. 2009;25(4):319-327. doi:10.1093/heapol/czp063

23. Kobusingye OC, Hyder AA, Bishai D, Hicks ER, Mock C, Joshipura M. Emergency medical systems in low- and middle-income countries: recommendations for action. Bull World Health Organ. 2005;83:626-631.

24. Infrastructure and Regional Integration Technical Working Group. Overview of the Transport Infrastructure Sector in the Kingdom of Cambodia. Ministry of Public Works and Transport; 2015. Accessed August 21, 2018. https://data.opendevelopm entmekong.net/dataset/8b056306-0cd1-4b71-8361-6 5da79adcfd0/resource/37398c0b-74cc-4bb8-a7cb-cc0 491059706/download/iritwgoverviewofthetransportin frastructuresectorinthekingdomofcambodia2015.pdf

25. Ministry of Planning. National Strategic Development Plan 2014-2018. Phnom Penh, Cambodia: Royal Government of Cambodia. Accessed September 17, 2018. https://cambodia.unfpa.org/en/publication s/national-strategic-development-plan-2014-2018

26. Phong K, Srou L, Sola J. Mobile Phones and Internet Use in Cambodia 2016. Open Institute; 2016. Accessed October 18, 2017. http://www.open.org.kh/research/p hones_2016.pdf

27. Ministry of Health. Clinical Practice Guideline for Paediatric. Kingdom of Cambodia Ministry of Health; 2013.

28. Ministry of Health. Clinical Practice Guideline for Medicine. Kingdom of Cambodia Ministry of Health; 2013.

29. National Reproductive Health Program. Safe Motherhood Clinical Management Protocols - Referral Hospital. Kingdom of Cambodia Ministry of Health; 2013.

30. Quality Health Services Project. Quality Health Services Emergency Care and Referral Guidelines. URC Co. LLC; 2016.
31. Krasovec K. Auxiliary technologies related to transport and communication for obstetric emergencies. Int J Gynaecol Obstet. 2004;85:S14-S23. doi:10.1016/j.ijgo.2004.02.007

32. Mucunguzi S, Wamani H, Lochoro P, Tylleskar T. Effects of improved access to transportation on emergency obstetric care outcomes in Uganda. Afr J Reprod Health. 2014;18:87-94.

33. Acker P, Newberry JA, Hattaway L (Bud) F, Socheat P, Raingsey PP, Strehlow MC. Implementing an Innovative Prehospital Care Provider Training Course in Nine Cambodian Provinces. Cureus. Published online June 27, 2016. doi:10.7759/cureus.656

34. Vermeir P, Vandijck D, Degroote S, et al. Mutual perception of communication between general practitioners and hospital-based specialists. Acta Clin Belg. 2015;70(5):350-356. doi:10.1179/2295333715y.0 000000032

35. Gandhi TK, Sittig DF, Franklin M, Sussman AJ, Fairchild DG, Bates DW. Communication breakdown in the outpatient referral process. J Gen Intern Med. 2000;15(9):626-631. doi:10.1046/j.1525-1497.2000.91 119. $\mathrm{x}$

36. Akbari A, Mayhew A, Al-Alawi MA, et al. Interventions to improve outpatient referrals from primary care to secondary care. Cochrane Database Syst Rev. Published online October 8, 2008. doi:10.100 2/14651858.cd005471.pub2

37. World Health Organization, ed. Handbook IMCI: Integrated Management of Childhood Illness. WHO; 2005.

38. Vargas I, Garcia-Subirats I, Mogollón-Pérez AS, et al. Understanding communication breakdown in the outpatient referral process in Latin America: a crosssectional study on the use of clinical correspondence in public healthcare networks of six countries. Health Policy Plan. 2018;33(4):494-504. doi:10.1093/heapol/c $\underline{\mathrm{zy} 016}$

39. Ramanayake RPJC. Structured printed referral letter (form letter); saves time and improves communication. J Family Med Prim Care. 2013;2(2):145. doi:10.4103/2249-4863.117404

40. Jenkins S, Arroll B, Hawken S, Nicholson R. Referral letters: are form letters better? Br J Gen Pract. 1997;47:107-118.

41. Huot E. Guideline for Referral System in Cambodia. Cambodia Ministry of Health; 2006. 
42. Nsibande D, Doherty T, Ijumba P, et al. Assessment of the uptake of neonatal and young infant referrals by community health workers to public health facilities in an urban informal settlement, KwaZulu-Natal, South Africa. BMC Health Serv Res. 2013;13(1):47. doi:10.1186/1472-6963-13-47

43. Siddiqi S. The effectiveness of patient referral in Pakistan. Health Policy Plan. 2001;16(2):193-198. do i:10.1093/heapol/16.2.193

44. Josyula S, Taylor KK, Murphy BM, Rodas D, Kamath-Rayne BD. Obstetric referrals from a rural clinic to a community hospital in Honduras. Midwifery. 2015;31(11):1054-1059. doi:10.1016/j.mid w.2015.07.002
45. National Institute of Statistics. Cambodia. Cambodia Inter-Censal Population Survey 2013. Phnom Penh. Ministry of Planning; 2013. Accessed November 5, 2017. http://www.stat.go.jp/info/meetings/cambodi a/pdf/ci fn02.pdf

46. United Nations, Department of Economic and Social Affairs-Population Division. World Population Prospections: The 2015 Revision, Key Findings and Advance Tables. United Nations; 2016. 